\title{
Visual field size necessary for length comparison
}

\author{
MITSUO IKEDA, SHINYA SAIDA, and TAKASHI SUGIYAMA \\ Department of Information Processing, Tokyo Institute of Technology, Nagatsuta \\ Midori-ku, Yokohama 227, Japan
}

\begin{abstract}
Influence of visual field size upon acuity for comparing lengths of two lines was investigated. The visual field was effectively narrowed to any desired size by use of a TV display system. The TV screen presented a portion of the test stimulus, the position of which was controlled by the subject's eye movement so that his fixation point always coincided with the center of the portion. The test stimulus was composed of two lines arranged horizontally, one of which had a length of $81 \mathrm{~mm}$ and the other a slightly different value. Sizes of the narrowed visual field were $20,40,70,100,150$, and $340 \mathrm{~mm}$ wide. The comparison acuity dropped rapidly as the visual field was narrowed to and below $70 \mathrm{~mm}$ which was about the same extent as one of the two lines. The implication is that our excellent ability for length comparison is only possible when we can observe the whole line at one time.
\end{abstract}

Good visual acuity occurs only in the central part of the retina with poor acuity in the periphery. Detail detection tasks are almost entirely carried out by the fovea in cooperation with appropriate eye movements, and it is generally thought that the periphery is not useful in such detail detection. In our daily experience, however, the peripheral retina functions continually. It detects various incoming stimuli and directs the fovea to them for further investigation if needed. Some research has been carried out on the extent of the functional visual area for the detection of incoming stimuli (Engel, 1971; Ikeda \& Takeuchi, 1975; Leibowitz \& Appelle, 1969; Sanders, 1970).

An important role of the peripheral field seems to be concerned with pattern recognition. Mooney (1957) showed that pictures were as effectively perceived with a single fixation, whether that fixation was brief or prolonged. The implication is that the peripheral visual field must be useful in pattern perception. This usefulness was directly demonstrated by artificially restricting the size of the visual field. Watanabe (1971) showed that pattern recognition became very difficult and even impossible if the peripheral visual field was not used. Similar deterioration occurred in reading with a narrowed visual field (Ikeda \& Saida, 1977; McConkie \& Rayner, 1975). These results all indicate that the peripheral visual field must function in order for the visual system to perceive patterns.

Our ability to compare the lengths of two horizontal lines presented simultaneously, one on the

T. Sugiyama is presently at Minolta Camera Research Laboratory, Daisen Nishimachi, Sakai, Osaka. right and one on the left, is very good, involving only $1 \%$ or $2 \%$ error when certain conditions are satisfied (see Le Grand, 1967, for review, and Pollock and Chapanis, 1952, for an example). We can detect a mere $1-\mathrm{mm}$ increment if we are looking at a line of $100-\mathrm{mm}$ length. In view of the fovea-centered characteristics of our visual system, we might consider such excellent ability to be due only to the fovea. However, the determination of line length is a form of pattern perception which makes use of the peripheral visual field. Buchsbaum (1972) measured fixation points of the subject's eye while engaged in comparing the sizes of two squares. One subject performed the task merely by looking at several points in the centers of the two squares. No fixation points fell upon the edges of the squares, which presumably would have occurred if the fovea were used exclusively for the task. The same may be true for length comparison. Our excellent ability in length comparison may be possible only by utilizing the peripheral retina.

It is uncertain, however, precisely how much of the visual field is necessary to assure accurate performance. We have developed a method of controlling the size of the visual field (Ikeda \& Saida, 1977) which is appropriate to this question. A subject is presented with two horizontal lines and asked to decide which is longer. His visual field is narrowed to a certain size and its influence upon the accuracy of comparison is investigated.

A supplementary experiment was conducted to investigate the influence of the time delay of presentation of lines, one from the other, upon the accuracy of length comparison. This is called the delayed presentation experiment. 


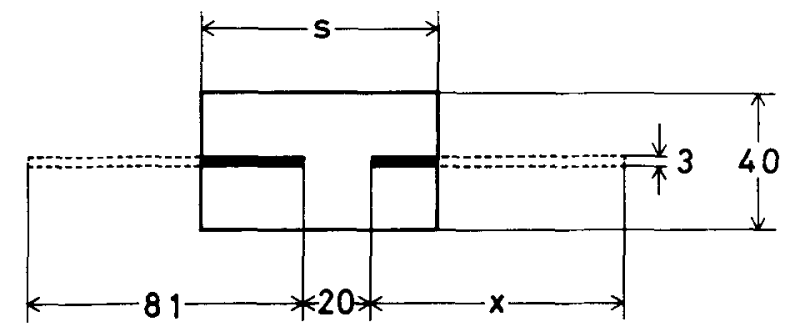

Figure 1. Dimensions of test stimulus and narrowed visual field in millimeters.

\section{METHOD}

\section{Apparatus and Stimuli}

Narrowed field. From a distance of $1 \mathrm{~m}$, the subject, with his right eye covered completely, looked at a $39 \times 31 \mathrm{~cm}$ TV monitor screen with his left eye. His head was stabilized with a bite board. The test stimulus, shown on the screen, was composed of two horizontal, right and left, lines with a gap between (see Figure 1). However, the test stimulus was not shown entirely at any one time. Only the part within a rectangular form was visible.

The horizontal position of the subject's eye was registered by the corneo-sclera reflection method and the data were fed into the TV monitor in order to control the horizontal position of the rectangular portion. The fixation point and the horizontal center of the rectangle were made to coincide precisely both during fixation and during eye movement. Thus, the subject's visual field was limited to that portion within the rectangular form, which we shall call the narrowed visual field. The vertical size of the narrowed visual field was fixed to $40 \mathrm{~mm}\left(2^{\circ} 18^{\prime}\right.$ arc of visual angle), and only the horizontal size was changeable. In the present experiment, we employed six sizes of $s=20,40,70,100,150$, and $340 \mathrm{~mm}$ or $1^{\circ} 9^{\prime}, 2^{\circ} 18^{\prime}, 4^{\circ}, 5^{\circ} 43^{\prime}, 8^{\circ} 32^{\prime}$, and $18^{\circ} 47^{\prime}$ arc of visual angle.

Dimensions of the test stimulus, as displayed on the screen, are given in Figure 1, together with the rectangular window that provided the narrowed visual field. It happens to be $s=70 \mathrm{~mm}$ in this instance. Two black lines were arranged horizontally $20 \mathrm{~mm}$ apart. The length of the left line was fixed to $80 \mathrm{~mm}\left(4^{\circ} 38^{\prime}\right.$ arc visual angle), while the other, $x$, was variable. We presented 20 different stimuli with $x=71,72,73, \ldots, 89,90$, and $91 \mathrm{~mm}$, excluding $x=81 \mathrm{~mm}$. The brightness of the dark line inside the narrowed visual field was $0.2 \mathrm{~cd} / \mathrm{m}^{2}$, while that of the immediate surroundings was $2.3 \mathrm{~cd} / \mathrm{m}^{2}$. The portion outside the narrowed visual field was uniformly dark with a luminance of $0.1 \mathrm{~cd} / \mathrm{m}^{2}$ in the case of subjects M.I., T.S., and T.W., while it was $2.3 \mathrm{~cd} / \mathrm{m}^{2}$, namely the same brightness as the inside of the narrowed visual field, in the case of subjects K.D., A.Z., and K.T. The latter condition was introduced to minimize the edge appearance of the narrowed visual field so that subjects could not utilize it as a cue for length estimation. The results, however, showed no difference between the two conditions.

Delayed presentation. A three-channel tachistoscope was used to present two horizontal lines with various time delay, $t$. The first channel provided one line on the left for a duration of $100 \mathrm{msec}$ and the second provided the other line on the right for the same duration but with the time delay, $t$. The time delays employed were $\mathrm{t}=0,0.5,1,2$, and $4 \mathrm{sec}$, the zero condition corresponding to the simultaneous presentation of both lines. The third channel merely provided a uniform white field whenever the first or the second channel was off. The duration of $100 \mathrm{msec}$ was determined experimentally to be the shortest duration providing as good length comparison accuracy as any longer duration.

Dimensions of the test stimulus were the same as in the case of the narrowed field experiment, in terms of visual angle. The subject's viewing distance was $85 \mathrm{~cm}$. Therefore, the line length corresponding to $81 \mathrm{~mm}$ in the previous experiment was $68.9 \mathrm{~mm}$ in this experiment. We shall refer to it as $81 \mathrm{~mm}$, however, in order to avoid unnecessary complication in reporting. The same applied to the other lengths also. The length of one of the two lines, either on the left or on the right, corresponded to $81 \mathrm{~mm}$ and the other corresponded to $71,72, \ldots$, or $91 \mathrm{~mm}$, excluding $81 \mathrm{~mm}$, providing 40 different stimuli. Lines were drawn black on white cardboard to be illuminated in the tachistoscope. The field brightness was $3.0 \mathrm{~cd} / \mathrm{m}^{2}$ throughout. The subjects used a headrest but no bite board. They observed the stimuli binocularly.

\section{Procedure}

Narrowed field. Before each experiment, the subject's fixation point and the horizontal center of the narrowed visual field were brought together by adjusting the eye position in relation to the photodiodes which registered the reflected lights from the sclera of the left eye. Normally it required about $30 \mathrm{~min}$ for a satisfactory adjustment. Throughout this period, the subject became accustomed to the apparatus and to the feeling of a narrowed visual field.

Next, 1 of 20 line lengths was chosen at random and prepared for presentation on the TV screen by means of a TV camera. The visual field of $s=340 \mathrm{~mm}$ was also prepared. Nothing appeared yet on the screen. When the experimenter said, "Start," the subject pressed a starting key and the narrowed visual field appeared on the screen. The subject was asked to detect which line in the test stimulus was longer. Eye movement was freely allowed and was recorded on a T-Y recorder for further analysis. As soon as the subject had made his decision, he released the starting key, and both the narrowed visual field and the test stimulus immediately disappeared. The subject, without disengaging himself from the bite board, reported "right" or "left" by means of a signal buzzer. The test stimulus was then changed and the same procedure repeated. All 20 stimuli were. explored successively in random order. Two subjects, M.I. and T.S., were aware that the left line was $81 \mathrm{~mm}$ throughout, but others T.W., K.D., A.Z., and K.T., were not informed that this length was constant

The visual field was then narrowed to $\mathrm{s}=150 \mathrm{~mm}$ and an experiment similar to $s=340 \mathrm{~mm}$ was carried out. Experiments, with $\mathrm{s}=100,70 \mathrm{~mm}$, and so forth, followed, completing the first experimental session. The second experimental session was conducted on another day in which the visual field of $s=$ $20 \mathrm{~mm}$ was first investigated, followed by $\mathrm{s}=40 \mathrm{~mm}$, and so on, to $s=340 \mathrm{~mm}$. Third and fourth sessions were held with some subjects, number of observations totaling 60 (subject T.S.) and 80 (other subjects).

Delayed presentation. Subjects fixated halfway between two fixation points placed vertically at the middle of the space between the two test lines. They pressed a button for the stimulus presentation. The left line always appeared first except for $t=0$. The subjects were required to maintain fixation until both lines were exposed. They then reported "right" or "left" vocally for the longer line. The stimuli were presented successively in random order until all 40 stimuli were tested for a given time delay, $t$. The 81-mm line appeared on every presentation, either on the left or on the right, but the subjects were not informed that this was the case.

Similar test sessions were repeated for the other three time delays. The number of observations provided by each subject totaled 160 over all time delays.

\section{Subjects}

Six subjects, all university students except M.I., were employed for the narrowed field experiment. Two male subjects, M.I. and T.S., were experienced in this kind of experiment, while the four female subjects had never served as subjects before in any psychophysical experiment. All had normal visual acuity of better than 1 , when measured with the Landolt's $c$ rings, with eyeglass correction in some subjects. 
Two females, K.D. and A.Z., further served as subjects for the delayed presentation experiment.

\section{RESULTS AND DISCUSSION}

\section{Narrowed Field}

Two examples of eye movements during the inspection of test stimulus $\mathrm{x}=79 \mathrm{~mm}$ are given in Figure 2: (a) for the visual field size $s=340 \mathrm{~mm}$, and (b) for $s=20 \mathrm{~mm}$. The ordinate gives the time scale increasing downward, the interval between two successive lines corresponding to $0.5 \mathrm{sec}$. The recording paper was driven at $1 \mathrm{~cm} / \mathrm{sec}$. The abscissa gives the horizontal positions of fixation points. Locations of two test lines are indicated on the abscissa. $T_{1}$ and $T_{2}$ at the extreme left indicate the on and off instances of the starting key which was operated by the subject. Hence he observed the stimulus from the time $T_{1}$ through $T_{2}$, or from $P_{1}$ to $P_{2}$ on the traces.

The records in Figure 2 show the general characteristics of eye movement, namely, fixations and saccades between them. When the visual field is large, Figure $2 \mathrm{a}$, the decision was completed in the very short period of about $0.7 \mathrm{sec}$. The subject fixated at only two or three points, indicating the use of a wide region of the retina for the size estimation. When the visual field was narrowed to a very small size, Figure $2 b$, the eye movement became systematic. The eye scanned the test stimulus step by step, start-
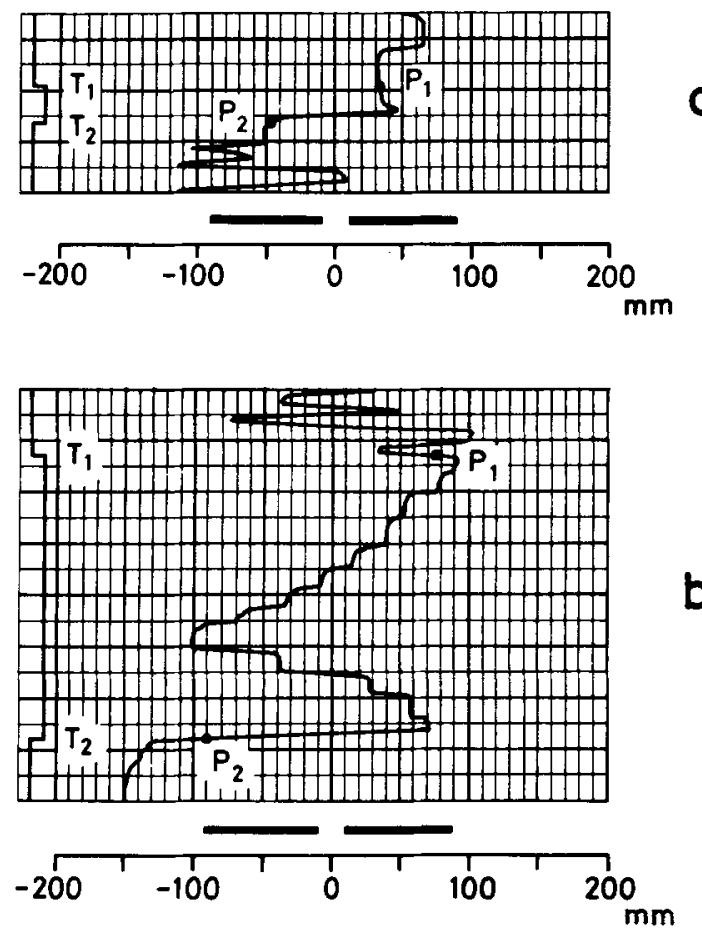

Figure 2. Eye-movement records of subject M.1., comparing lengths of two lines with narrowed visual fields: (a) $\mathrm{s}=340 \mathrm{~mm}$, (b) $\mathrm{s}=20 \mathrm{~mm}$. The subject observed the test stimulus from $P_{1}$ through $P_{2}$. ing at one end and ending at the other end. Some subsequent scanning followed. The saccadic movement in the first scanning approximately corresponded to the size of the visual field employed. This may indicate that, with the handicap of no peripheral visual field, the subject utilized the saccadic movement as a scale to measure the line length. Time needed for the decision increased to, in this instance, $5.4 \mathrm{sec}$. Yet the subject often was incorrect in his decision.

Incorrect responses, when plotted as a function of $x$, naturally occur more often at values of $x$ near $81 \mathrm{~mm}$, and we may assume the distribution to be Gaussian. We can calculate the standard deviation by using the Probit analysis and define it as the threshold of the length comparison, denoted by $\Delta \mathrm{L}$. Thresholds are summarized in Table 1 and plotted in Figure 3a for individual subjects and in Figure $3 \mathrm{~b}$ for the means. The size of visual fields, $\mathrm{s}$, is taken along the abscissa in the logarithmic scale and the threshold $\Delta \mathrm{L}$ along the ordinate. The relative threshold, namely $\Delta L / L$, is shown along the right ordinate, where $\mathrm{L}$ is taken as $81 \mathrm{~mm}$.

Although the threshold values vary among subjects, particularly at narrow visual fields, the influence of the narrowing visual field is quite apparent. Thresholds increase very rapidly as the visual fields get narrower. The influence is relatively small with subject T.S., probably because he was the most experienced subject for the present experiment and had gained some kind of strategy to overcome the handicap of his narrowed visual field.

We may recall that subjects M.L. and T.W. were provided with distinct borders at the edges of the visual fields while subjects K.D., A.Z., and K.T. were not. There seems to be no systematic difference in the results between these two groups, as seen in Table 1 or in Figure 3a. We may, therefore, assume that subjects did not utilize the visible visual field edges as the length reference. This was also confirmed by the subjective impression of the subjects.

In Figure $3 \mathrm{~b}$, we notice that the threshold is small and constant for visual fields wider than $100 \mathrm{~mm}$. It becomes clearly larger when the field is narrowed to $70 \mathrm{~mm}$ and gets even worse for still narrower visual fields. The critical visual field size, denoted by $s_{c}$, for assuring good acuity is about $100 \mathrm{~mm}$, implying that size estimation of a line is optimal as long as the visual field covers the line entirely. For good line length estimation, observation of a line as a whole, not part by part, is necessary.

The mean thresholds for visual fields narrower and wider than the critical field size appear to lie on two straight lines (Figure 3b) and can be approximated by the following equations.

$$
\Delta \mathrm{L} / \mathrm{L}=-0.097 \log \mathrm{s}+0.222 \text { for } \mathrm{s}<\mathrm{s}_{\mathrm{c}}
$$



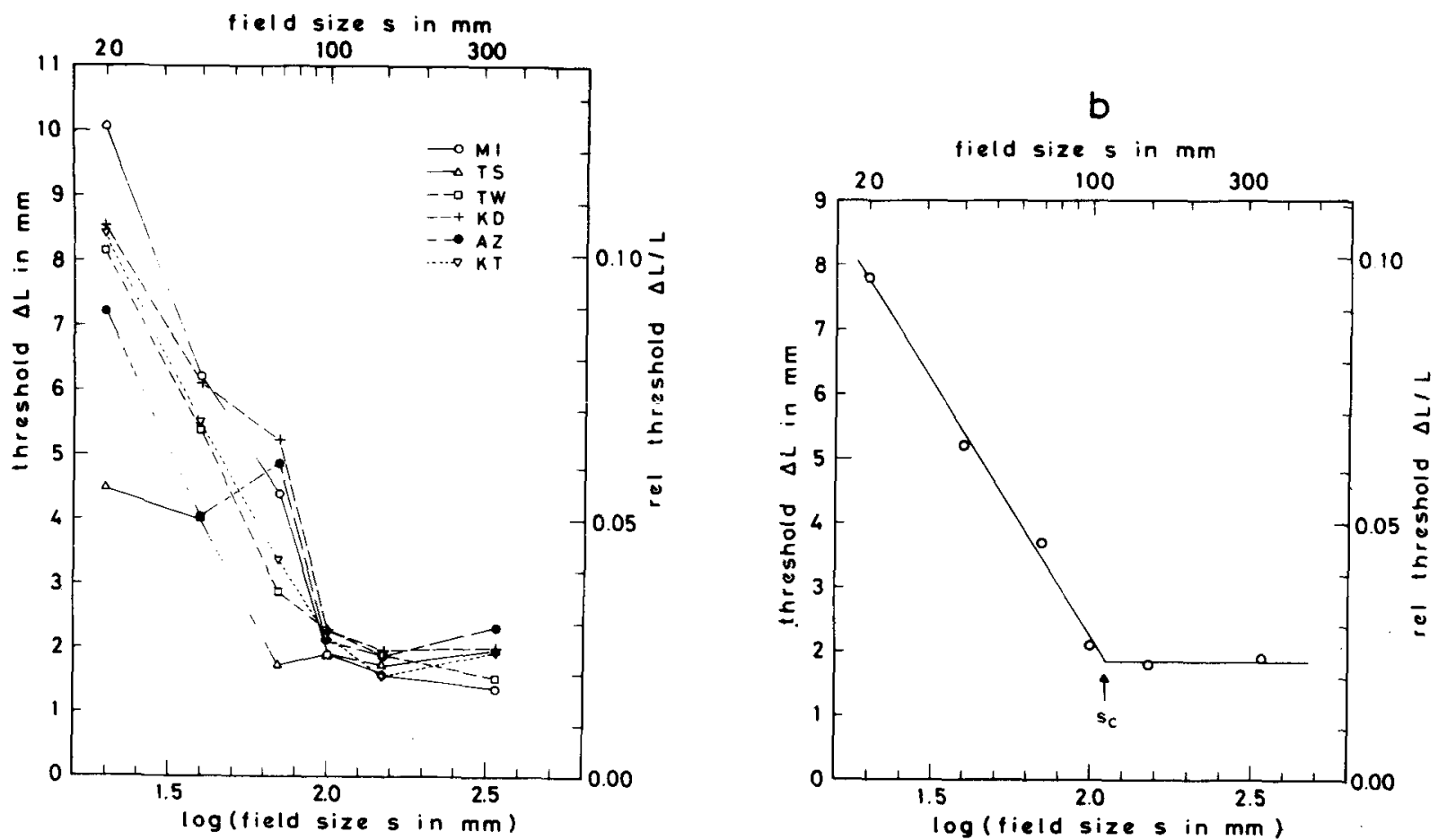

Figure 3. Thresholds for comparison of two line lengths as a function of narrowed visual field size: (a) plots of individual data, (b) means.

$$
\Delta \mathrm{L} / \mathrm{L}=0.023 \quad \text { for } \mathrm{s}>\mathrm{s}_{\mathrm{c}} \text {, }
$$

where $\mathrm{s}_{\mathrm{c}}=112 \mathrm{~mm}$ or $\log \mathrm{s}_{\mathrm{c}}=2.05$. The equations are strictly valid only for $\mathrm{L}=81 \mathrm{~mm}$.

The visual process producing such a straight line function as the first equation is not immediately obvious, and undue emphasis should not be attached to the value 0.023 in the second equation. This value can easily be manipulated by altering the observing conditions. In our case, the subject viewed the stimulus monocularly rather than binocularly. The stimulus was a TV-displayed figure which could not be made free from blur. The luminance of the stimulus was relatively low to avoid the afterimage of the

Table 1

Threshold $\Delta \mathbf{L}$ for Line Length Comparison $(81 \mathrm{~mm}$ vs. $\mathbf{x} \mathrm{mm}$ ), Defined by the Standard Deviation of the Incorrect Response Distribution in Millimeters

\begin{tabular}{lrrrrrr}
\hline & \multicolumn{5}{c}{ Visual Field Size s in Millimeters } \\
\cline { 2 - 7 } Subjects & \multicolumn{1}{c}{20} & 40 & 70 & 100 & 150 & 340 \\
\hline M.I. & 10.1 & 6.2 & 4.4 & 1.9 & 1.6 & 1.4 \\
T.S. & 4.5 & 4.0 & 1.7 & 1.9 & 1.7 & 2.0 \\
T.W. & 8.1 & 5.4 & 2.9 & 2.3 & 1.9 & 1.5 \\
K.D. & 8.5 & 6.1 & 5.2 & 2.3 & 2.0 & 2.0 \\
A.Z. & 7.2 & 4.1 & 4.9 & 2.1 & 1.9 & 2.3 \\
K.T. & 8.4 & 5.5 & 3.4 & 2.2 & 1.5 & 1.9 \\
Mean & 7.8 & 5.2 & 3.7 & 2.1 & 1.8 & 1.9 \\
\hline
\end{tabular}

rectangular visual field on the TV screen. These conditions may have all acted to reduce acuity and to give a rather large value of the relative threshold, 0.023 .

To analyze eye-movement behavior while subjects were engaged in the comparison task, we measured the locations of the fixation points on the records of the T-Y recorder. These are presented as histographs with $11-\mathrm{mm}$ intervals in Figure 4 for subjects M.I. and T.W. The abscissa indicates positions along the test stimulus, zero corresponding to the center of the gap between the two lines. The ordinate gives the frequencies of fixations at respective positions. In cach figure, the average number of fixations for each stimulus is indicated. When a subject was provided with a large visual field, a small number of fixation points were used. Subject T.W. tended to fixate the central portion of the stimulus when the field size was $340 \mathrm{~mm}$, while subject M.I. fixated the stimulus at random. For a visual field of 150,100 , or $70 \mathrm{~mm}$, the histographs show two distinct peaks, indicating that the fixation points fall around the middle portions of the respective lines. This distribution implies that the entire line was included within the visual field as much as possible and that it is necessary to observe the whole line at once in order to estimate the line length accurately.

With $s=40$ or $20 \mathrm{~mm}$, the fixation points again 

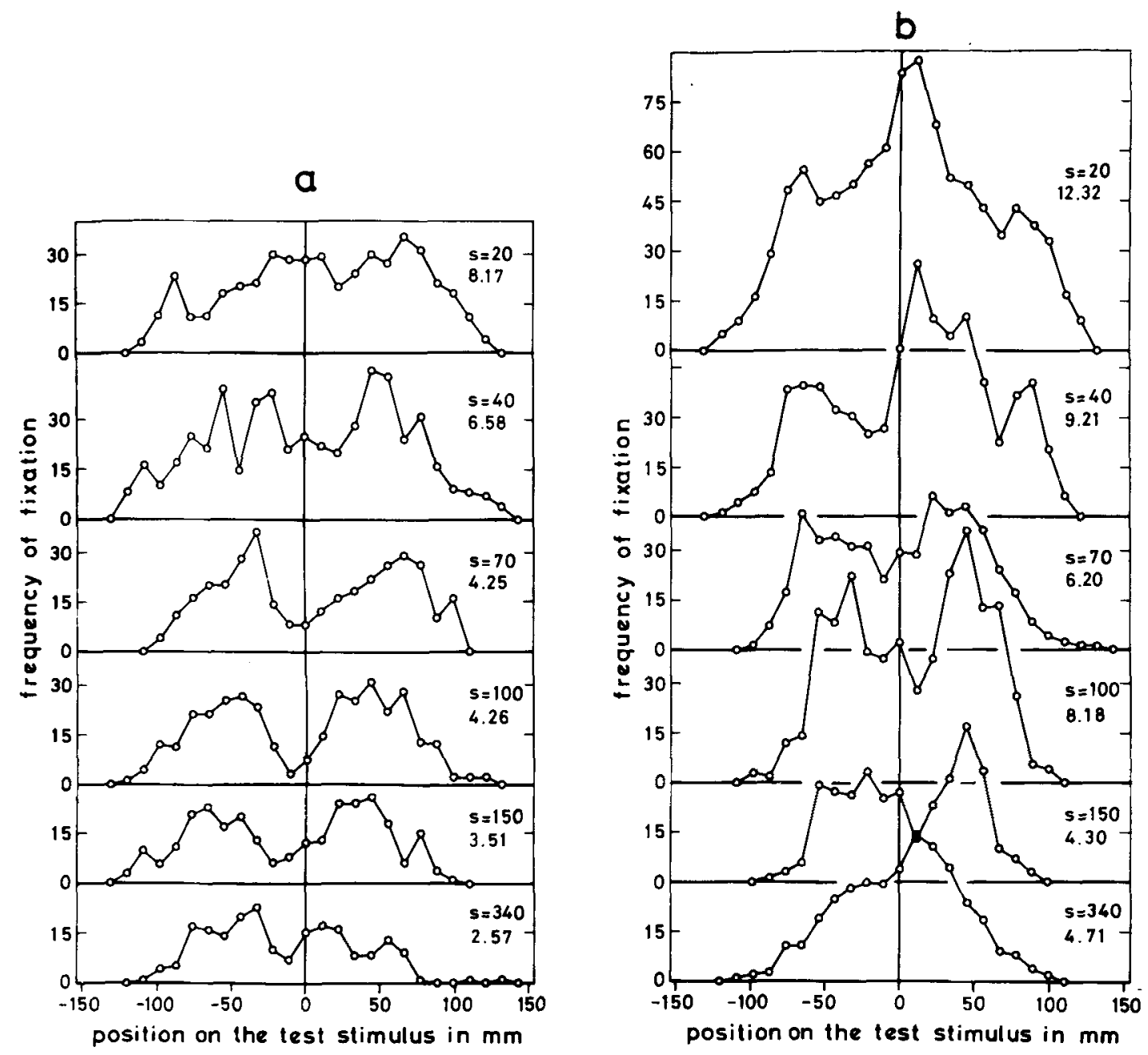

Figure 4. Histographs showing frequencies of fixation points at locations over the test stimulus when subjects engaged in line length comparison with narrowed visual fields, $s=20,40,70,100,150$, and $340 \mathrm{~mm}$. Average number of fixations at one observation is given in inset. Each figure is based on 80 observations of test stimulus. (a) Subject M.I., (b) subject T.W.

scattered all over the test stimulus and the average number of fixations increased. This pattern indicates that the subjects scanned the entire test stimulus with their narrow visual fields in order to estimate the line lengths. This is most clearly shown in Figure $2 b$. The mechanism for line-length estimation is no longer that of pattern perception as such, but is probably based on the eye movement itself. Accuracy was very poor, and the subjects found the task difficult.

Another aspect obtainable from the eye-movement traces in relation to the delayed presentation experiment is the time lag between observing one line and the other, which is inevitably introduced with narrower visual fields. As we saw in Figure $2 b$, the length comparison is achieved by the eye scanning over the stimulus from one end near $P_{1}$ to the other end, requiring about $3.6 \mathrm{sec}$. We may consider that the length estimation for the right line was mostly completed at the halfway point of scanning between $P_{1}$ and the first left end and that estimation of the left line was completed at the first left end. Therefore, the time lag between the two successive estimations is approximated as $1.8 \mathrm{sec}$ in the present instance, or half the total scanning time over the stimulus from one end to the other. This time lag was equivalent to the time delay introduced tachistoscopically in the delayed presentation experiment.

Table 2 summarizes the time lag obtained from eye movements of subjects K.D. and A.Z., using half of their entire eye-movement records. Clear eye scanning over the two stimulus lines was observed only for visual fields narrower than $\mathrm{s}=100 \mathrm{~mm}$. Therefore, the analysis was not conducted for $\mathrm{s}=$ 150 and $340 \mathrm{~mm}$. 
Table 2

Time Lag in Seconds Between Observing the Two Test-Stimulus Lines

\begin{tabular}{ccccc}
\hline & \multicolumn{4}{c}{ Visual Field Size s in Millimeters } \\
\cline { 2 - 5 } Subjects & 20 & 40 & 70 & 100 \\
\hline K.D. & 1.7 & 1.3 & 1.2 & .8 \\
A.Z. & 2.2 & 1.5 & 1.3 & 1.3 \\
\hline
\end{tabular}

\section{Delayed Presentation}

Threshold $\Delta$ Ls were obtained, as in the case of the narrowed visual field experiment, by calculating the standard deviation of the normal distribution best fitting the incorrect responses with the help of Probit analysis. The threshold values for subjects K.D. and A.Z. are shown in Figure 5 as a function of the time delay $t$ between line presentations.

The smallest thresholds occurred at $\mathrm{t}=0$ and $0.5 \mathrm{sec}$, that is, at simultaneous, or very close, presentations of both lines. The threshold value, however, appears slightly larger than the value for $\mathrm{s}=340 \mathrm{~mm}$ in Figure $3 \mathrm{a}$. This is probably due to the restriction on free eye movement and to the duration of stimulus presentation.

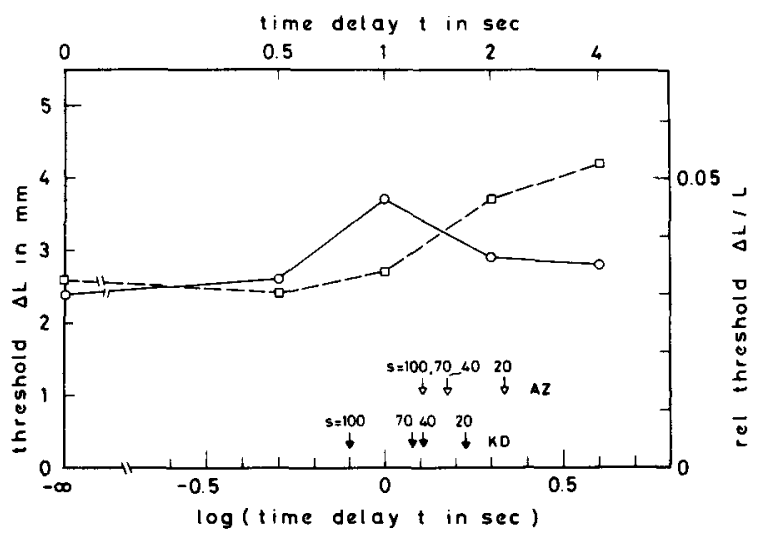

Figure 5. Thresholds for comparison of two line lengths as a function of time delay between the two lines.
For larger delays, the threshold increased gradually for subject A.Z. but no systematic increase occurred for subject K.D. In Figure 5, the time lags obtained in the previous section (Table 2) are indicated by arrows. The increase in the threshold due to the increase of the time delay from $s=100$ to $20 \mathrm{~mm}$ in the case of subject A.Z. was found to be less than $1.0 \mathrm{~mm}$ and was probably zero in the case of K.D. These negligible values eliminate the time lag observed in the narrowed field experiment as a possible cause for increasing the threshold when the visual field was narrowed to $20 \mathrm{~mm}$. The conclusion derived in the previous section, that the observation of a line as a whole is essential for good line length estimation, is thus supported.

\section{REFERENCES}

Buchsвaum, M. Individual differences in eye-movement patterns. Perceptual and Motor Skills, 1972, 35, 895-901.

ENGEL, F. L. Visual conspicuity, directed attention and retinal locus. Vision Research, 1971, 11, 563-576.

IKEDA, M.. \& SAIDA, S. Span of recognition in reading. Vision Research, 1977, 17, in press.

IKEDA, M., \& TAKEUCHI, T. Influence of foveal load on the functional visual field. Perception \& Psychophysics, 1975, 18. 255-260.

Le Grand, Y. [Form and space vision] (M. Millodot \& G. G. Health, Trans.). Bloomington: Indiana University Press, 1967.

Leibowitz, H. W., \& Appelle, S. The effect of a central task on luminance thresholds for peripherally presented stimuli. Human Factors, 1969, 11, 387-392.

McConkme, G. W., \& RAYNER, K. The span of the effective stimulus during a fixation in reading. Perception \& Psychophysics, $1975,17,578-586$.

Mooney, C. M. Closure as affected by viewing time and multiple visual fixations. Canadian Joumal of Psychology, 1957, 11 . 21-28.

Pollock, W. T., \& Chapanis, A. The apparent length of line as a function of its inclination. Quarterly Joumal of Experimental Psychology, 1952, 4, 895-901.

SANDERs, A. F. Some aspects of the selective process in the functional visual field. Ergonomics, 1970, 13, 101-107.

WatanaBe, A. Fixation points and eye movements. Oyobutsuri. 1971, 40, 330-334. (In Japanese)

(Received for publication March 16, 1977; revision accepted May 21, 1977.) 